\title{
VIH/SIDA: COMPORTAMIENTO EPIDEMIOLÓGICO DE LA TRANSMISIÓN VERTICAL EN EL CONTEXTO GENERAL DE LA INFECCIÓN EN CHILE
}

\author{
Enrique Valdés.$^{1}{ }^{1}$, Alvaro Sepúlveda M. ${ }^{2}$, Paula Candia P. ${ }^{3}$, Carolina Sepúlveda B. ${ }^{a}$ \\ Karinna Lattes $A{ }^{2}$ \\ ${ }^{1}$ Unidad de Medicina Materno Fetal, Hospital Clínico José Joaquín Aguirre, Universidad de Chile, ${ }^{2}$ Programa en Obstetricia y \\ Ginecología, Universidad de Chile. ${ }^{3}$ Académica Universidad Mayor.
}

a Alumna, Escuela de Medicina, Universidad de Chile.

\section{RESUMEN}

Antecedentes: En embarazadas seropositivas sin profilaxis antirretroviral la transmisión vertical (TV) del $\mathrm{VIH}$ es de $30 \%$, cifra que disminuye bajo al $2 \%$ con un manejo integral de prevención. Objetivo: Conocer el comportamiento epidemiológico de la TV en Chile desde la creación del programa nacional de prevención. Método: Datos aportados por el Departamento de Epidemiología del MINSAL y CONASIDA (1984-2006). Estudió de situación VIH/SIDA por año, regiones, grupo etario y sexo, con especial énfasis en TV. Resultados: Se notificaron 9.317 casos de VIH y 7.886 casos de SIDA (1984-2006), prevalencia en aumento hasta el 2003 con tendencia descendente posteriormente. La razón actual de VIH entre hombres y mujeres es de 4:1, con clara tendencia al aumento de notificación en mujeres. La principal vía de exposición sigue siendo la vía sexual $(93,4 \%)$. El grupo más afectado está entre los $20-39$ años $(73,1 \%$ para $\mathrm{VIH}$ y $63,1 \%$ para SIDA), cabe destacar que entre $0-9$ años se encuentra el $1,1 \%$ de los afectados por VIH y el 1,4\% por SIDA, todos infectados por TV. Conclusiones: La transmisión vertical de VIH en Chile es responsable de un bajo porcentaje de las personas notificadas de VIH/SIDA, pero es la causa de la totalidad de los niños afectados por la infección en nuestro país. Se observa una importante reducción de la TV, llegando a niveles muy cercanos a los objetivos ministeriales.

\section{PALABRAS CLAVE: VIH, SIDA, transmisión vertical, embarazo}

\section{SUMMARY}

Background: The risk of transmitting HIV from mother to unborn child, without any antiretroviral prophylaxis, reaches $30 \%$. It can be reduced to less than $2 \%$ by implementing integral preventive strategies. Objective: To assess the epidemiological profile of HIV vertical transmission in Chile since the implementation of the national AIDS transmission prevention program. Method: Data from the Epidemiology Department of the Health Ministry and the National Commission for AIDS grouped by year, location, age and gender. With special emphasis on vertical transmission. Results: 9317 cases of HIV infection and 7886 cases of AIDS have been reported in Chile (1984-2006), with a decreasing tendency since 2003. Sexual exposure continues to be the primary route with $93.4 \%$ of the cases. The male/female ratio for case notification is $4: 1$, with a rising tendency among women. The most affected is between $20-39$ years old $(73 \%$ HIV notification and $63.1 \%$ for AIDS). Among children between $0-9$ years of age is $1.1 \%$ of the HIV and $1.4 \%$ of AIDS notifications, all of them for vertical transmission. Conclusions: HIV vertical transmission in Chile is responsible for a small percentage of HIV/AIDS notifications, but it accounts for all of the infected children. There's been an important reduction in this transmission route, reaching prevalence close to the aimed rates, which can explained by the expanding prevention programs of the health policies implemented by the Health Ministry. 


\section{INTRODUCCIÓN}

El virus de la inmunodeficiencia humana $(\mathrm{VIH})$ pertenece a la familia retrovirus, de la subfamilia lentivirus, del cual se han identificado dos tipos: $\mathrm{VIH}-1$, el más frecuente, que conduce al síndrome de inmunodeficiencia adquirida (SIDA) y a la muerte; VIH-2, principalmente en habitantes o visitantes del África Occidental, transmitido con menor eficacia, más indolente, pero que también termina en SIDA y muerte. Ambos tipos son de estructura viral y genómica muy similar. En América Latina, reportes del año 2006 muestran que el número estimado de personas seropositivas es de 1,7 millones, con 140.000 casos nuevos y 65.000 fallecidos, destacando el hecho que dos terceras partes de los infectados viven en cuatro países; Argentina, Brasil, Colombia y México (1).

En Chile, la epidemia se ha caracterizado por el sostenido aumento de la portación principalmente de VIH 1 en mujeres en edad fértil por lo que las estrategias de salud diseñadas para disminuir el riesgo de transmisión vertical (TV) están destinadas a programas de detección, prevención y de tratamiento. Es así que, en nuestro país la vigilancia epidemiológica del VIH/SIDA se realiza mediante la notificación obligatoria (mecanismo pasivo) y a través de estudios de seroprevalencia de VIH con metodología de Centro Centinela (mecanismo activo), desarrollados en Chile desde el año 1992 (2). La Comisión Nacional del SIDA (CONASIDA) es un organismo perteneciente al Ministerio de Salud, creado el 4 de mayo de 1990, y que cumple con el fin de coordinar y evaluar todas las actividades relacionadas con estrategias de prevención y tratamiento del VIH/SIDA en Chile (3). El protocolo de prevención de la transmisión Vertical (PPTV) fue aprobado oficialmente por Decreto Oficial el 17 de octubre de 2005, considerando el manejo de la embarazada $\mathrm{VIH}+$ en tres etapas: durante el embarazo tratamiento con terapia antirretroviral (TARV) desde las 24 semanas; durante el parto, con TARV 4 horas antes del trabajo de parto; y al recién nacido, con TARV las primeras seis semanas de vida (3). Previo al año 2005 se utilizaba el esquema ACTG 076, el cual administraba Zidovudina durante el embarazo, el parto y en el recién nacido hasta las seis semanas de vida.

El objetivo de este estudio es conocer el comportamiento epidemiológico de la transmisión por VIH/SIDA en la mujer chilena desde la creación del programa ministerial de prevención.

\section{MÉTODO}

Se estudió el comportamiento epidemiológico y el manejo de los pacientes portadores de VIH/SIDA en Chile desde el comienzo de la epidemia hasta los últimos registros notificados al Departamento de Epidemiología del Ministerio de Salud y CONASIDA (enero de 1984 - diciembre de 2006). Se estudió la situación de $\mathrm{VIH} / \mathrm{SIDA}$ por año, regiones, grupo etario y sexo, colocando especial énfasis en la transmisión en la población femenina.

\section{RESULTADOS}

En Chile, el total de casos notificados de portadores del VIH y de aquellos que padecen SIDA es de 9.317 y 7.886 respectivamente, entre los años 1984 y 2006 (Figura 1). Las regiones que presentan las mayores tasas de notificación de seropositivos son la primera, metropolitana y la quinta, con una razón de 115,5, 96,4 y 79,1 por 100.000 habitantes, respectivamente (2). En estas mismas regiones, las tasas de SIDA notificadas corresponden a 92, 85,1 y 65,6 por 100.000 habitantes, respectivamente (2). En los primeros años, la razón de casos notificados de VIH entre hombres y mujeres era de 11:1, actualmente esta razón es de 4:1 lo que demuestra una clara tendencia al aumento de nuevos portadores en la población femenina, correspondiendo este grupo a un $15 \%$ de las notificaciones de VIH/SIDA (4) (Figura 2). El número total de mujeres portadoras de VIH/SIDA en los últimos 8 años es de 2.707 lo que corresponde al $18,7 \%$ del total de la población afectada (Figura 3). El grupo etario más afectado se encuentra entre los 20 y 39 años $(73,1 \%$ para VIH y $63,1 \%$ para SIDA).

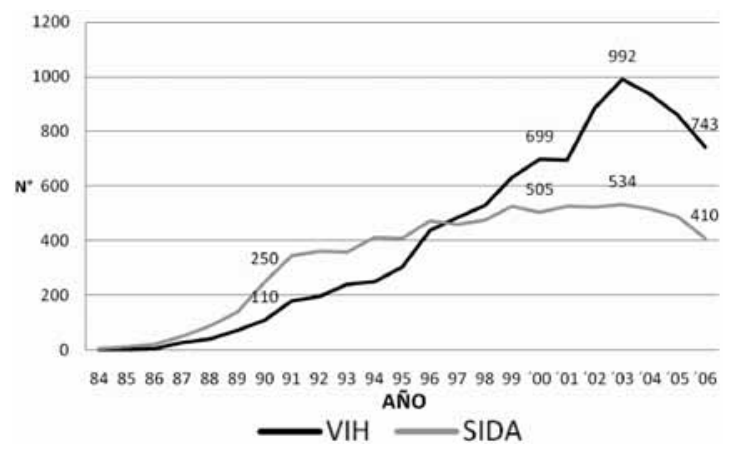

Figura 1. Total de casos VIH/SIDA notificados por año (Chile 1984-2006). Fuente: referencia 4. 


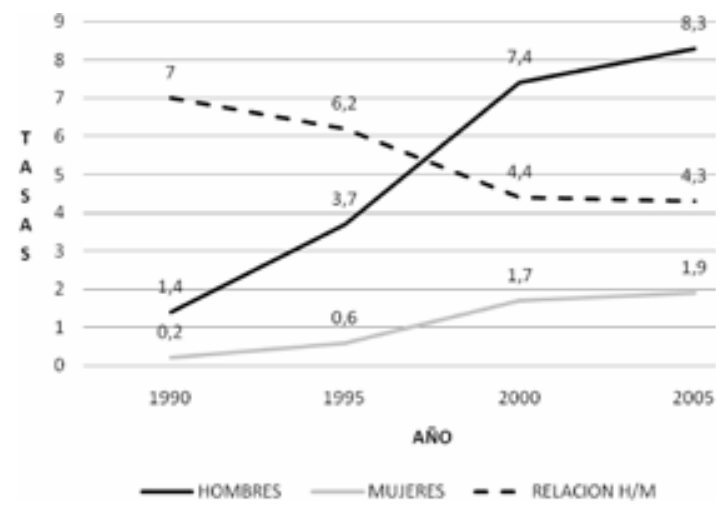

Figura 2. Notificación VIH por sexo, Chile 19902005 (tasas $\times 100$ mil habitantes). Fuente: referencia 3.

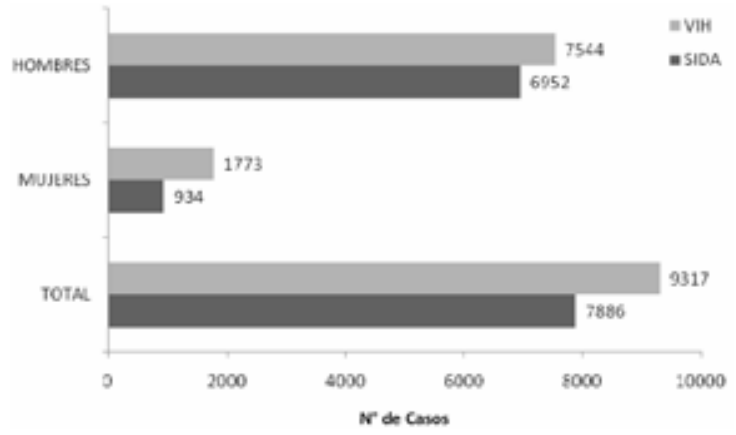

Figura 3. Total de casos notificados por sexo y diagnóstico, desde 1998 al 31 diciembre 2006. Fuente referencia 4.

Respecto a la vía de transmisión del virus la principal vía de exposición sigue siendo la sexual que concentra el $93,4 \%$ de los casos, correspondiendo un $34,8 \%$ a heterosexuales, grupo que se incrementó de un 13,4\% entre los años 1986-1990 a un $24 \%$ entre los años 2001-2005, siendo a su vez el grupo más frecuente dentro de la población femenina, con un $74 \%$ de las afectadas (4).

La vía vertical es responsable del $1,1 \%$ de los casos de $\mathrm{VIH}$ y el $1,4 \%$ de los casos de SIDA durante los años 1998 al 2006, lo que concuerda con el rango etario de 0 a 9 años (Figuras 4,5 y 6). Al analizar este punto por distribución por sexo, se puede apreciar que durante el período 1986-2005 la infección por VIH/SIDA por TV en niñas es de un 3,2\%, mientras que en niños es de sólo un $0,6 \%$ (4).

La situación de la mortalidad por SIDA desde 1990 a 2004, información rescatada del registro de los certificados de defunción de ese período, dan cuenta que entre 1990 y 2004 se produjeron en el país 4.893 defunciones por SIDA, de las cuales el $12 \%$ correspondieron a mujeres. Según los últimos indicadores básicos de salud disponibles en Chile, en el año 2005 la mortalidad por VIH/SIDA ocupó el octavo lugar en ambos sexos, con una tasa de 2,4/100 mil habitantes (correspondiendo un 4,1 a hombres y 0,7 a mujeres) (5) (Figura 7).

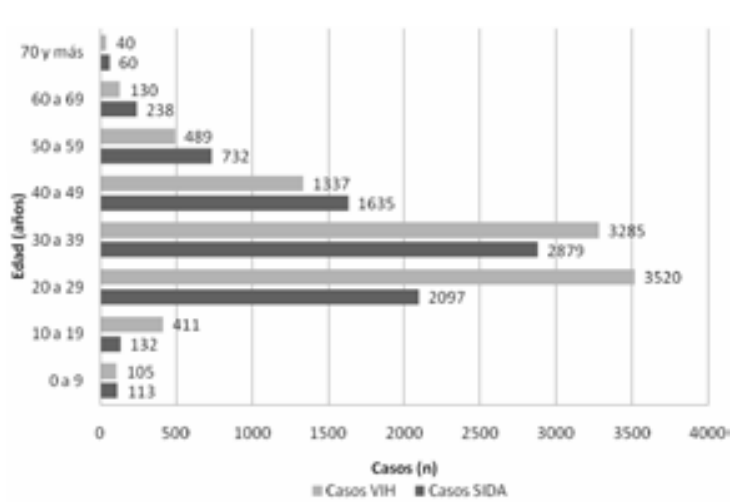

Figura 4. Casos notificados de VIH/SIDA por rango de edad, Chile 1998-2006. Fuente: referencia 4.

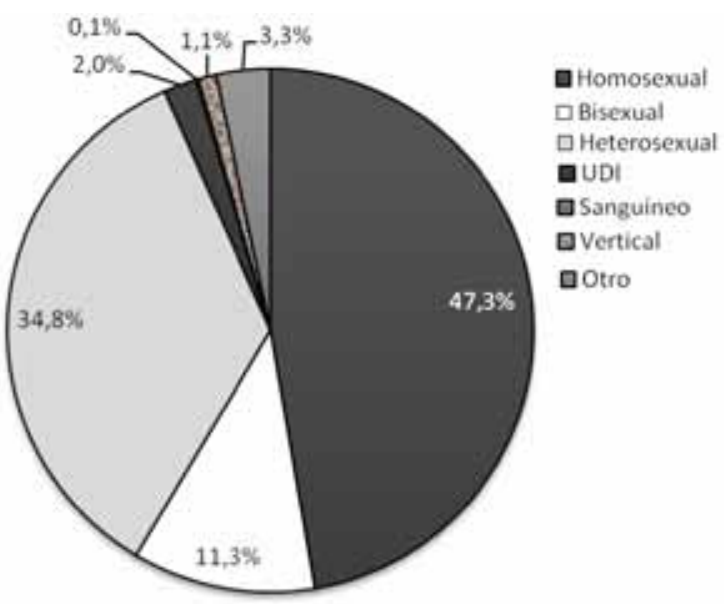

Figura 5. Total de personas notificadas de VIH según vía de exposición al virus. ( $n=9.317$ casos), (UDI: uso de drogas inyectables). Fuente: referencia 4. 


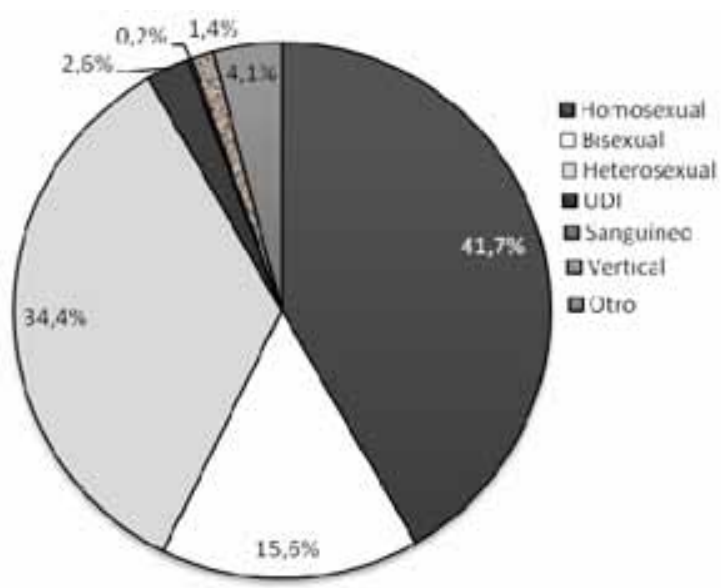

Figura 6. Total personas notificadas de SIDA según vía de exposición. ( $\mathrm{n}=7.886$ casos), UDI: uso drogas inyectables. Fuente: referencia 4.

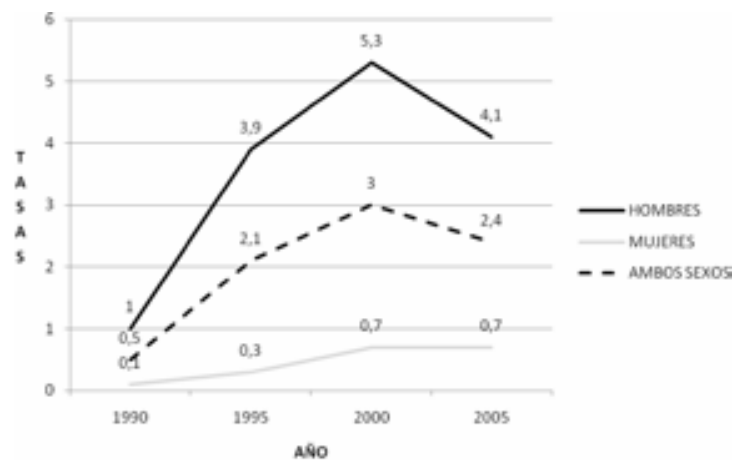

Figura 7. Mortalidad por VIH/SIDA, Chile 19902005 (tasas $\times 100$ mil habitantes). Fuente: DEIS 2006 y 2007 , MINSAL.

\section{DISCUSIÓN}

En Chile, la prevalencia de VIH/SIDA en la población general aumentó hasta el año 2003 para posteriormente observarse una sostenida tendencia a la baja, tendencia explicada por el exitoso programa ministerial de prevención y tratamiento, no pudiéndose excluir la falta de notificación de casos nuevos.

Existen razones que explican el por qué la mujer es más susceptible de adquirir la infección por VIH que el hombre, por lo que el personal de salud debe ser consciente de dichos factores y así poder educar a la población como estrategia de prevención de la infección. Dentro de estos se deben co- nocer: A) la transmisión sexual del virus es varias veces mayor del hombre a la mujer que viceversa, presentando mayor riesgo aquellas mujeres que presenten una mucosa vaginal adelgazada o desprovista de mecanismos fisiológicos de defensa (púberes, posmenopaúsicas) (6); B) las vaginosis, muchas veces asintomática por lo que frecuentemente no son diagnosticadas, alteran la indemnidad de la mucosa vaginal (6-8); C) la dependencia al hombre que presenta la mujer en nuestro país en lo económico, social y emocional, hacen de la mujer más proclive de infectarse debido a la dificultad de rechazar a tipos de relaciones sexuales de alto riesgo (6-8); D) pautas culturales que alientan la promiscuidad en el varón, lo que aumente el riesgo de contagio en las monógamas (6-8) y E) falta de fácil acceso a métodos preventivos controlados por la mujer (preservativos femeninos) (6-8).

Probablemente por estas razones, es que la proporción de mujeres portadoras del VIH en Chile ha aumentando con relación a los hombres, debido que la principal vía de contagio es la sexual $(93,6 \%)$ esto sumado al impacto de la vía de transmisión del virus en forma homo y bisexual $(46,1 \%)$, transforma a la mujer monógama heterosexual como la población de mayor riesgo $(51,1 \%)$. Así mismo, existe un aumento sostenido a través del tiempo de casos contagiados por relaciones entre hombres y mujeres en comparación con los transmitidos en forma homosexual, desplazándose la epidemia hacia grupos poblacionales heterosexuales, con mayor impacto en el grupo poblacional femenino (2).

Por lo antes expuesto es posible concluir que la caracterización de la epidemia en Chile ha variado, tendiendo a la heterosexualización y feminización ( $18,7 \%$ del total de la población afectada). Por otro lado, el hecho que el grupo etario más afectado se encuentre entre los 20 y 39 años $(73,1 \%$ para $\mathrm{VIH}$ y $63,1 \%$ para SIDA) es de mucha importancia, ya que demuestra que la población mayormente afectada es la correspondiente a la de edad fértil, situación que influye directamente en el riesgo de la TV. Lo que avala la importancia de la educación de la sexualidad humana a todo nivel poblacional, proporcionando la información necesaria y reconociendo el derecho de la mujer a informarse, para así lograr que ella tome las medidas apropiadas para protegerse y prevenir la infección por el $\mathrm{VIH}(9,10)$.

Con relación a la serotipología del VIH 1 , un reciente estudio concluyó que el genotipo más frecuentemente aislado en la población chilena fue el subtipo B en 202 casos (91,4\%), con distribución amplia a través de todo el país, seguido por el subtipo $\mathrm{F}$ en 19 casos $(8,6 \%)$ encontrado principalmente el centro y norte de la nación $(p<0,01)$, subtipo 
aislado en mujeres que adquirieron la infección por contacto heterosexual y niños infectados por TV y que al parecer fue introducido al territorio a partir del contagio con habitantes provenientes de los otros países del cono sur americano (Argentina, Brasil y Uruguay) (11).

Estudios epidemiológicos sugieren que las tasas de embarazos en mujeres seropositivas que no han presentado SIDA son comparables con aquellas no infectadas, mientras que las que desarrollaron la enfermedad la probabilidad de embarazarse es bastante menor. Por otra parte, el $90 \%$ de las mujeres $\mathrm{VIH}+$ conocieron su estado de portación durante el embarazo y puerperio, al pesquisarse la infección en su descendencia, por lo que adquiere relevancia el conocer la condición de portación en la consulta pre y/o posconcepcional, ya que existe suficiente evidencia de la efectividad del tratamiento durante el embarazo para disminuir el riesgo de TV. La serología para VIH se aconseja realizarla a toda mujer en edad fértil previa "consejería" y consentimiento informado. A este respecto cabe señalar que en nuestro país, según la normativa legal vigente (resolución exenta $N^{\circ} 371$ del 2 de febrero de 2001 publicada en el diario oficial el día 9 de febrero de 2001), obliga al acto de consejería pretest, donde un personal debidamente entrenado informa a la paciente las implicancias médicas, sociales legales y éticas del estudio, la cual voluntariamente decide realizarse o no el examen. Esto ha demostrado ser muy importante en la toma de conciencia de la enfermedad, adhesividad al tratamiento y la adquisición de conductas en la portadora que eviten la transmisión a su entorno personal (12-14).

Afortunadamente, el estado gravídico no parece alterar la evolución o la historia natural de la infección por $\mathrm{VIH}$, ya que si bien es cierto se ha descrito un descenso del recuento de células CD4 durante el embarazo, estas vuelven a su línea basal después del parto (15). En ausencia de complicaciones, tales como drogadicción o enfermedad médica crónica, la incidencia de patología obstétrica no aumenta, permaneciendo el aborto espontáneo, la restricción del crecimiento intrauterino, el parto de pretérmino y la muerte fetal, dentro de prevalencias esperables (8). Sin embargo, en gestantes con un compromiso significativo del sistema inmunitario (CD4 <300/dl) el riesgo de las complicaciones infecciosas se incrementan (16).

El enfrentamiento clínico a una gestante seropositiva se debe basar en una evaluación minuciosa de su estado de salud inicial, realizándose un completo examen físico, dando especial atención a aquellos signos que nos orienten a patología infecciosa oportunista y evaluando su estado inmunitario actual (6).
Los casos de VIH y SIDA notificados por TV alcanzan al $1,1 \%$ y $1,4 \%$ respectivamente, la que sin profilaxis antirretroviral sería de un $29 \%$, estimándose una incidencia de seropositividad en la población gestante chilena de un 0,5/1000 embarazadas, lo que significaría esperar una cifra aproximada de 100 gestantes VIH + por año (6). En Chile, desde 1994 se han implementado diversos protocolos para la prevención de la TV, que han incluido: utilización de terapia antiretroviral durante el embarazo, parto y al recién nacido, cesárea programada dependiendo de la carga viral (17) y suspensión de la lactancia materna, con lo que se ha logrado una importante disminución de TV. Sin embargo, la norma ministerial establece que toda mujer portadora de VIH debe ser interrumpida vía cesárea. Con este objetivo a partir de 1996 se ha intentado disminuir el riesgo de TV utilizando un manejo clínico terapéutico antiretroviral con el objetivo de lograr cargas virales maternas bajas o indetectables sin efectos tóxicos y/o teratogénicos fetales. El primero de ellos, ACTG 076 consistía en la administración de Zidovudina durante el embarazo y el parto, como también al recién nacido hasta las seis semanas de vida, indicando operación cesárea electiva y contraindicando la lactancia materna. Este esquema logró disminuir la TV desde un $29 \%$ a un $5,6 \%$ en el año 2001(18-20). Posteriormente, debido a la aparición de nueva evidencia que sostenía que el tratamiento triasociado era más efectivo que la monoterapia en prevenir la TV, se diseñó un protocolo utilizando una combinación de inhibidores de transcriptasa reversa y de proteasas, esquemas denominados en su conjunto como terapia retroviral de alto grado de actividad (TARGA). Gracias a esta, la TV ha descendido a $1,1 \%$ cifra próxima a alcanzar el objetivo trazado en el año 2005 de reducirla a un 1\%.

Por último, la estrategia de prevención de la TV se ha basado en la continua revisión de la evidencia acumulada, por lo que es de importancia seguir evaluando nuevas conductas tendientes a identificar en otros aspectos: la eventual inducción de resistencia y toxicidad de los antiretrovirales en la gestante como en el recién nacido y su potencial efecto en la calidad de vida posterior, la utilización de micronutrientes y su impacto en disminuir la TV, la indicación de la operación cesárea en gestantes con baja carga viral y el lavado espermático como elemento reductor de TV (21-25).

\section{CONCLUSIÓN}

Chile es uno de los dos países con los mejores índices de control de la epidemia VIH/SIDA en Latino América. La TV de VIH es responsable de un 
bajo porcentaje de las personas notificadas de $\mathrm{VIH} /$ SIDA, pero es la causa de la totalidad de los niños afectados por la infección en Chile. Se ha visto una importante reducción de la transmisión por esta vía de exposición, llegando a niveles muy cercanos a los objetivos propuestos, posiblemente explicada por la amplia cobertura y efectividad de las medidas preventivas propuestas por las autoridades de salud.

AGRADECIMIENTOS: Ofrecemos nuestros agradecimientos a la Sra. Gloria Berríos, enfermera encargada del Programa Transmisión Vertical VIH/SIDA CONASIDA, por su colaboración en el aporte de información fundamental para el desarrollo de esta publicación.

\section{BIBLIOGRAFÍA}

1. UNAIDS, 2006. Situación de la Epidemia de América. Hallado en: www.unaids.org. Acceso el 01/09/2007.

2. Ministerio de Salud de Chile. CONASIDA. Boletín de Epidemiología $\mathrm{N}^{\circ} 12$ y 13, 2000. Hallado en: www. minsal.cl. Acceso el 01/09/2007.

3. Ministerio de Salud de Chile. CONASIDA. Estrategia de atención integral a personas que viven con $\mathrm{VIH} /$ SIDA. Programa Nacional de Prevención y Control del VIH/SIDA, 2006;1-13. Hallado en: www.minsal.cl. Acceso el 01/09/2007.

4. Departamento de Epidemiología, DIPLAS-MINSAL. Evolución del VIH-SIDA Chile, 1986-2005; 1-14. Hallado en: http://webhosting.redsalud.gov.cl/minsalarchivosvih/Pais_86-05/vihsidaepi.pdf. Acceso el 01/09/2007.

5. MINSAL. Indicadores Básicos de Salud, Chile 2007. Hallado en: http://deis.minsal.cl/deisindicadores/ indi2007.pdf. Acceso el 01/09/2007.

6. Herane E, Valdés E. Mujer y VIH. En: Sepúlveda C, Afani A. SIDA. Santiago. Editorial Mediterráneo, $2^{\circ}$ Edición, 1997;438-56

7. Valdés E. VIH-SIDA y embarazo: actualización y realidad en Chile. Rev Chil Obstet Ginecol 2002;67:160-6.

8. Valdés E. VIH-SIDA y embarazo. En: García Huidobro M, Hasbún J, (ed). Urgencias y complicaciones en obstetricia. Editorial Mediterráneo. Santiago. 2006;73-83.

9. de Bruyn M, Paxton S. HIV testing of pregnant women--what is needed to protect positive women's needs and rights? Sex Health 2005;2:143-51.

10. Dhai A, Noble R. Ethical issues in HIV. Best Pract Res Clin Obstet Gynaecol 2005;19:255-67.

11. Rios M, Fernandez J, Jaramillo $P$, Paredes V, Sanchez JL, Laguna-Torres VA, Carr JK, Ramirez E. Molecular epidemiology of HIV type 1 in Chile: Differential geographic and transmission route distribution of $B$ and $F$ subtypes. AIDS Res Hum Retroviruses 2005;21:835-40.

12. Bowden FJ. Reconsidering HIV testing--consent is still the key. Sex Health 2005;2:165-7.

13. Chou R, Smits AK, Huffman LH, Fu R, Korthuis PT; US Preventive Services Task Force. Prenatal screening for HIV: A review of the evidence for the U.S. Preventive Services Task Force. Ann Intern Med 2005;143:38-54.

14. Campos-Outcalt D. Time to revise your HIV testing routine. J Fam Pract 2007;56:283-4.

15. Burns DN, Nourjah $P$, Minkoff $H$, Korelitz J, Biggar RJ, Landesman S, Rubinstein A, Wright D, Nugent RP. Changes in CD4+ and CD8+ cell levels during pregnancy and post partum in women seropositive and seronegative for human immunodeficiency virus-1. Am J Obstet Gynecol 1996;174:1461-8.

16. Minkoff HL, Willoughby A, Mendez H, Moroso G, Holman S, Goedert JJ, Landesman SH. Serious infections during pregnancy among women with advanced HIV infection. Am J Obstet Gynecol 1990;162:30-4.

17. Abarzúa F, Pérez $C$, Callejas $C$, Yombi JC, Vandercam B. Ausencia de transmisión perinatal de $\mathrm{VIH}$ en 40 embarazadas tratadas con terapia anti-retroviral de alta potencia. Rev Chil Obstet Ginecol 2004;69:232-8.

18. Connor EM, Sperling RS, Gelber R. Reduction of maternal-infant transmission of human inmunodeficiency virus type 1 with zidovudine treatment. N Engl J Med 1994;331;18: 1173-80.

19. Ministerio de Salud de Salud de Chile, CONASIDA. Evaluación de protocolo ACTG 076 en Chile. Boletín de Epidemiología $N^{\circ} 12$. Disponible en: www.minsal. cl. (consulta 01/09/2007).

20. Read JS, Newell MK. Efficacy and safety of cesarean delivery for prevention of mother-to-child transmission of HIV-1. Cochrane Database Syst Rev 2005;(4):CD005479.

21. Abarzua $F$, Nuñez $F$, Hubinont $C$, Bernard $P$, Yombi JC, Vandercam B. Human immunodeficiency virus (HIV) infection in pregnancy: antiretroviral treatment (ART) and mode of delivery. Rev Chil Infectol 2005;22:327-37.

22. Semprini AE, Vucetich A, Hollander L. Sperm washing, use of HAART and role of elective caesarean section. Curr Opin Obstet Gynecol 2004;16:465-70.

23. El Beitune P, Duarte G, Quintana SM, Figueiro-Filho EA, Marcolin AC, Abduch R. Antiretroviral therapy during pregnancy and early neonatal life: consequences for HIV-exposed uninfected children. Braz J Infect Dis 2004;8:140-50.

24. Poirier MC, Olivero OA, Walker DM, Walker VE. Perinatal genotoxicity and carcinogenicity of anti-retroviral nucleoside analog drugs. Toxicol Appl Pharmacol 2004;199(2):151-61.

25. Brogly SB, Ylitalo N, Mofenson LM, Oleske J, Van Dyke R, Crain MJ, Abzug MJ, Brady M. Jean-Philippe $P$. Hughes MD. Seage GR 3rd. In utero nucleoside reverse transcriptase inhibitor exposure and signs of possible mitochondrial dysfunction in HIV-uninfected children. AIDS 2007;21:929-38. 